\title{
A gravidez na adolescência e suas perspectivas biopsicossociais
}

\author{
Adolescent pregnancy and its biopsychosocial perspectives \\ El embarazo em la adolescencia y sus perspectivas biopsicosociales
}

Paulo Guilherme Alves Gonzaga ${ }^{1 *}$, Carla Anatália Aparecida de Araújo Pereira ${ }^{1}$, Bruna de Souza Costa $^{1}$, Camila Késsia Nogueira Silva ${ }^{1}$, Bruna Martins dos Santos ${ }^{1}$, Roberta Ribeiro Souto ${ }^{1}$, Fernando de Andrade Pinheiro', Débora Florisbelo de Castro Barbosa ${ }^{1}$, Laís Vieira de Lima1, Mariana Rossi Silva da Paixão'.

\section{RESUMO}

Objetivo: Abordar os fatores epidemiológicos e sociodemográficos ligados a gravidez na adolescência. Métodos: Esta revisão integrativa teve como base um levantamento bibliográfico acerca da gravidez na adolescência. As etapas para sua construção foram: selecionar a temática, buscar artigos publicados entre os anos de 2011 e 2021 nas bases de dados digitais, estabelecer critérios de inclusão, exclusão, e discussão dos artigos, sendo selecionados ao final 16 artigos. Resultados: A taxa de fecundidade da população vem caindo, mas percebe-se que adolescentes entre 15 e 19 anos representam $23 \%$ da fecundidade nacional, sendo sua principal causa o nível de vulnerabilidade em que essas jovens estão expostas. Em relação aos riscos, apesar das divergências de autores, observa-se que a probabilidade de mortes nessa faixa etária em relação ao parto e à gravidez é duas vezes maior que em mulheres de 20 anos ou mais. As principais consequências são a evasão escolar, depressão, distúrbios emocionais e ideações suicidas. Considerações finais: A prevalência e o aumento da proporção de gravidez na adolescência ao longo dos anos é preocupante, sendo considerada um caso de calamidade pública no Brasil necessitando, assim, de intervenções no que tange, principalmente, a promoção de saúde.

Palavras-chave: Gravidez na adolescência, Hebiatria, Obstetrícia.

\begin{abstract}
Objective: to discuss epidemiological and demographic factors related to teenage pregnancy. Methods: this integrative literature review was based on a bibliographic search about teenage pregnancy. The stages of construction of this review were: selecting the theme, searching for articles published between 2011 and 2021 in digital databases, establishing criteria for inclusion/exclusion and discussion of articles, being selected at the end 16 articles. Results: The population fertility rate has been falling. Adolescents between 15 and 19 years old are $23 \%$ of the national fertility because of the socioeconomic vulnerability level at which these women are exposed. Regarding the risks, it is observed that the probability of deaths childbirth/pregnancy in this age group are twice higher as $20+$ aged women. Furthermore, the main consequences of teenage pregnancy are dropping out of school, depression, emotional disturbances and suicidal ideation. Final considerations: The prevalence and increase in the proportion of teenage pregnancy over the years is worrying, being considered a case of public calamity in Brazil, thus needing interventions with regard, mainly, to health promotion.
\end{abstract}

Keywords: Pregnancy in adolescence, Adolescent medicine, Obstetrics.

${ }^{1}$ Instituto Master de Ensino Presidente Antônio Carlos (IMEPAC), Araguari - MG.

*E-mail: pauloguilherme210330@hotmail.com 


\section{RESUMEN}

Objetivo: Abordar los factores epidemiológicos y sociodemográficos vinculados al embarazo adolescente. Métodos: Esta revisión bibliográfica integradora se basó en un estudio de la literatura sobre el embarazo adolescente. Los pasos en la construcción de esta revisión fueron: seleccionar el tema, buscar artículos publicados entre los años 2011 y 2021 en bases de datos digitales, establecer criterios de inclusión, exclusión y discusión de los artículos, siendo seleccionados al final 16 artículos. Resultados: La tasa de fecundidad de la población ha decrecido. Se da cuenta de que las adolescentes entre 15 y 19 años son en $23 \%$ de la fecundidad nacional debido al nivel de vulnerabilidad socioeconómica al que están expuestas estas mujeres. En cuanto a los riesgos, se observa que la probabilidad de parto/embarazo mortal en este grupo de edad es dos veces mayor que la de las mujeres de más de 20 años. Además, las principales consecuencias del embarazo adolescente son el abandono escolar, la depresión, los trastornos emocionales y la ideación suicida. Consideraciones finales: La prevalencia y el aumento de la proporción de embarazos en adolescentes a lo largo de los años es preocupante, considerándose un caso de calamidad pública en Brasil, needitando, assim, de intervenções no que tange, principalmente, una promoção de saúde.

Palabras clave: Embarazo en adolescencia, Medicina del adolescente, Obstetricia.

\section{INTRODUÇÃO}

Segundo a Sociedade Brasileira de Pediatria (SBP), a adolescência é uma etapa da vida que se singulariza pela transição da infância para a idade adulta. Essa etapa compreendida entre 10 e 20 anos incompletos é marcada por grandes transformações físicas, cognitivas e sociais que apresentam grande relevância para a concepção da identidade pessoal (SBP, 2019).

A relação sexual no período da adolescência, tem seu início cada vez mais precoce, com consequências indesejáveis imediatas, como o aumento da frequência de infecções sexualmente transmissíveis e gravidez precoce (MINISTÉRIO DA SAÚDE, 2017). A gestação nesse período é considerada como uma situação de tensão individual e risco social, visto que sua magnitude engloba adversidades como conflitos familiares, abandono do estudo e discriminação social (CASTRO ABC, et al., 2018; MARANHÃO TA, et al., 2018).

A gravidez na adolescência e suas complicações no pré-natal e parto são importantes causas de mortalidade entre adolescentes gestantes. No Brasil, entre as adolescentes de 10 a 14 anos, o índice de fecundidade permanece contínuo, porém entre as adolescentes de 15 a 19 anos constata-se que o índice está diminuindo (CABRAL CS e BRANDÃO ER, 2020). Estudos comprovam que baixa escolaridade e baixa renda são fatores de risco importantes para a gestação na adolescência(PINHEIRO YT, et al., 2019). Ressalta-se que a gravidez na adolescência continua sendo um problema de saúde pública (FREITAS MVP e SANTOS FR, 2020).

Em relação às complicações da gestação para a jovem, mencionam-se maiores incidências de anemia materna, desproporção céfalo-pélvica, síndrome hipertensiva da gravidez, infecção urinária, depressão pósparto, placenta prévia e complicações durante o trabalho de parto, acarretando aumento da mortalidade materna e infantil (BACELAR EB, et al., 2017, CABRAL ALB, et al., 2020).

No que tange o neonato, a gestação precoce está relacionada a taxas maiores de parto pré-termo e baixo peso ao nascer, além de maior frequência de intercorrências pré-natais, no trabalho de parto e no puerpério (FARIA GRB e MAGALHÃES CMC, 2020).

O presente estudo objetivou, por meio da análise de 16 artigos, observar os fatores que permeiam os riscos da gravidez na adolescência, pontuar quais os principais motivos que levam a gestação precoce, identificar as características das adolescentes mais vulneráveis e ressaltar quais são as principais consequências biopsicossociais da gestação na adolescência, bem como realizar uma análise sobre os fatores epidemiológicos e sociodemográficos relacionados a essa temática, criando um panorama da produção científica sobre os risco, causas e consequências da gravidez na adolescência. 


\section{MÉTODOS}

A pesquisa é uma revisão integrativa que analisa o conhecimento acerca dos dados publicados no Brasil em relação aos fatores epidemiológicos e sociodemográficos associados à gravidez na adolescência. Esta revisão é conduzida para identificar, analisar e sintetizar resultados de estudos independentes sobre o mesmo assunto, contribuindo para uma melhor compreensão acerca das perspectivas biopsicossociais e clínicas do tema (SOUZA MT, et al., 2010).

O processo do estudo, seguiu-se com a busca na literatura, determinando critérios em concordância ao objetivo da pesquisa, considerando os participantes, a intervenção e os resultados de interesses (SOUZA MT, et al., 2010). Os critérios de inclusão foram artigos publicados entre os anos de 2011 e 2021 para se ter maior abrangência do tema elencado já que períodos menores apresentaram escassez de trabalhos publicados, inseridos na base de dados Brasil Scientific Electronic Library Online (SciELO), na Biblioteca Virtual em Saúde (BVS) e na base de dados da Acervo+, com texto completo e de livre acesso, disponíveis na língua portuguesa e que abordam a gravidez na adolescência. Já os critérios de exclusão foram artigos de revisão, teses, dissertações, relatos de casos, artigos que abordassem a gravidez na fase adulta e também aqueles com deficiência metodológica.

A coleta de dados foi feita a partir da busca na base de dados SciELO, Acervo+ e BVS, que é composta pelas seguintes bases de dados: Literatura Latino-americana e do Caribe em Ciências da Saúde (LILACS), Medical Literature Analysis and Retrieval System Online (MEDLINE), Literatura do Caribe em Ciências da Saúde (MEDCARIB), Pan American Health Organization - Institutional Repository for Information Sharing (PAHO-ÍRIS) e World Health Organization Library Information System (WHOLIS).

Através dessas plataformas, houve a coleta dos artigos científicos aplicando os descritores em língua portuguesa "Gravidez na adolescência AND Riscos AND Causas AND Consequências" e em língua inglesa " Pregnancy in Adolescence AND Risk Factors AND Causality AND Consequence Analysis". A partir desta coleta, a variedade da amostra desta revisão integrativa em conjunto com as múltiplas finalidades desse método, proporciona resultados com conceitos complexos, de teorias ou problemas relativos ao foco do estudo (ERCOLE FF, et al., 2014).

A revisão desses artigos foi realizada através de uma leitura minuciosa, análise através da construção de uma tabela, contendo os autores e ano, objetivos, perfil metodológico e principais resultados, dividindo assim, a pesquisa, entre a epidemiologia da gravidez na adolescência, as causas, os riscos e as consequências psicossociais associadas ao tema.

\section{RESULTADOS}

O processo de seleção dos artigos encontrados após levantamento nas bases de dados Scielo, BVS e Acervo+, está sintetizado no fluxograma apresentado na Figura 1. Foram identificados 120 artigos, dos quais foram excluídos 42 por não abordarem a gravidez na adolescência, suas causas, riscos ou consequências. Outros 21 artigos foram excluídos por serem tese ou monografia, relato de caso, relato de experiência e artigo de revisão. E foram descartados mais 41 artigos por repetição. Dessa forma, foram selecionados 16 estudos para o desenvolvimento deste trabalho. 
Figura 1 - Seleção e coleta das publicações na amostra da revisão integrativa.

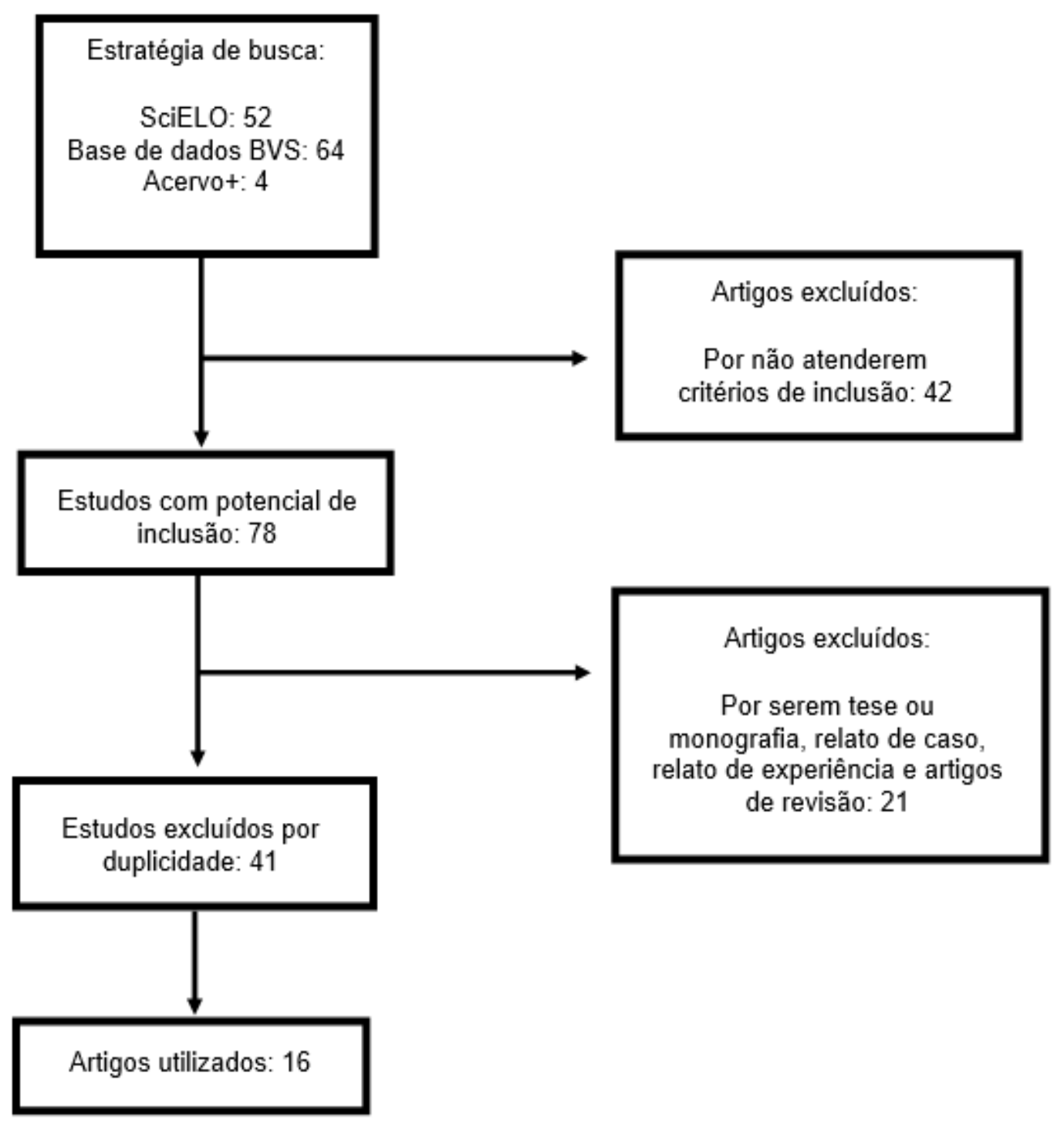

Fonte: Gonzaga PGA, et al., 2021.

Nos artigos selecionados, observa-se que o período temporal de maior publicação foram os anos de 2012, 2014 e 2019 com três publicações em cada, seguidos do ano de 2016 com duas e 2011, 2013, 2017, 2018 e 2020 com uma publicação por ano. Os anos de 2015 e 2021 não apresentaram nenhuma produção publicada nas bases de dados utilizadas.

O Quadro 1 mostra qual o perfil metodológico dos artigos selecionados. Nota-se que $62,5 \%$ destes fizeram uma análise qualitativa, 31,25\% realizaram abordagem quantitativa e 6,25\% tiveram teor misto. Já $43,75 \%$ conseguiram abordar as causas da gestação na adolescência, $31,25 \%$ os riscos e $18,75 \%$ as consequências psicossociais.

Percebe-se que, dos 16 artigos selecionados, 10 são de teor qualitativo ou de cunho observacional, descritivo ou analítico, o que revela a necessidade de realização de estudos que propõem intervenções para verificar as consequências positivas ou negativas e confirmar ou refutar hipóteses levantadas a partir de uma análise teórica desta temática. Apenas 5 dos estudos observados tiveram teor totalmente quantitativos e um apresentou teor misto, revelando a extrema importância de desenvolvimento de estudos que verifiquem as taxas de consequências que refletem na gestação precoce. Além disso, o mesmo quadro apresenta dados sobre o objetivo, o perfil metodológico e os principais resultados encontrados nos artigos selecionados. 
Quadro 1 - Dados sobre o objetivo, perfil metodológico e principais resultados dos artigos incluídos no estudo.

\begin{tabular}{|c|c|c|c|}
\hline Autores e Ano & Objetivos do estudo & Perfil metodológico & Principais resultados \\
\hline $\begin{array}{l}\text { TABORDA JA, et } \\
\quad \text { al., } 2014 .\end{array}$ & $\begin{array}{l}\text { Identificar e analisar as consequências objetivas e subjetivas da } \\
\text { gravidez na adolescência, considerando diferenças } \\
\text { socioeconômicas. }\end{array}$ & $\begin{array}{l}\text { Estudo exploratório } \\
\text { qualitativo. }\end{array}$ & $\begin{array}{l}\text { Maior impacto entre as famílias de baixa renda, principalmente quanto ao } \\
\text { adiamento ou comprometimento dos projetos educacionais, menor chance de } \\
\text { qualificação profissional e dependência financeira absoluta da família. }\end{array}$ \\
\hline $\begin{array}{l}\text { MIURA PO, et } \\
\text { al., } 2018 .\end{array}$ & $\begin{array}{l}\text { Compreender a experiência emocional decorrente da violência } \\
\text { intrafamiliar vivenciada pelas mães adolescentes e adolescentes } \\
\text { grávidas acolhidas institucionalmente. }\end{array}$ & $\begin{array}{l}\text { Estudo de caráter } \\
\text { exploratório, } \\
\text { descritivo e clínico- } \\
\text { qualitativo. }\end{array}$ & $\begin{array}{l}\text { A violência traz consequências na vida das adolescentes, intenso sofrimento, } \\
\text { e a repetição que atravessa gerações, demandando um cuidado de toda a } \\
\text { sociedade, na prevenção e intervenção. }\end{array}$ \\
\hline $\begin{array}{l}\text { KUDLOWIEZ S e } \\
\text { KAFROUNI R, } \\
2014 .\end{array}$ & $\begin{array}{l}\text { Compreender os projetos de vida de um grupo de adolescentes } \\
\text { grávidas, investigando os projetos antes de engravidarem e seus } \\
\text { planos futuros depois da gravidez se confirmar. }\end{array}$ & $\begin{array}{l}\text { Estudo qualitativo } \\
\text { e de campo. }\end{array}$ & $\begin{array}{l}\text { As adolescentes não demonstraram conhecimentos consistentes com } \\
\text { projetos de longo prazo, como estratégias ou caminhos a serem trilhados } \\
\text { para alcançar os objetivos pretendidos. }\end{array}$ \\
\hline $\begin{array}{l}\text { RIBEIRO CPS, } \\
\text { et al., } 2016 .\end{array}$ & $\begin{array}{l}\text { Descrever a percepção dos adolescentes quanto às } \\
\text { transformações corporais, riscos e consequências de uma } \\
\text { gravidez na adolescência, pautados na caderneta de saúde do } \\
\text { adolescente. }\end{array}$ & $\begin{array}{l}\text { Estudo descritivo e de } \\
\text { campo }\end{array}$ & $\begin{array}{l}\text { Observou-se que os adolescentes não conhecem a caderneta e demonstraram } \\
\text { o interesse em conhecer o conteúdo. }\end{array}$ \\
\hline $\begin{array}{l}\text { CARMO SS, et } \\
\text { al., } 2014 .\end{array}$ & $\begin{array}{l}\text { Verificar frequência, causas e consequências da gravidez na } \\
\text { adolescência. }\end{array}$ & $\begin{array}{l}\text { Estudo quantitativo e } \\
\text { descritivo. }\end{array}$ & $\begin{array}{l}\text { Concluiu-se ser necessária a aproximação com as adolescentes para } \\
\text { esclarecer dúvidas, desprender-se de preconceitos, realizar assistência } \\
\text { holística com orientações objetivas, corretas e claras, respeitando crenças e } \\
\text { individualidades. }\end{array}$ \\
\hline $\begin{array}{l}\text { GALLO JHS, } \\
2011 .\end{array}$ & $\begin{array}{l}\text { Analisar a ocorrência dos vários eventos simultâneos à gravidez } \\
\text { em adolescentes, as diferenças e heterogeneidade de acordo } \\
\text { com a idade das gestantes por ocasião do nascimento dos bebês } \\
\text { e, contribuir para uma reflexão sobre a gravidez na adolescência } \\
\text { e suas consequências e repercussões nas esferas individual, } \\
\text { familiar e social. }\end{array}$ & $\begin{array}{l}\text { Estudo transversal, } \\
\text { documental e } \\
\text { analítico. }\end{array}$ & $\begin{array}{l}\text { O estudo analisou que uma em cada três gestantes eram adolescentes, e } \\
\text { houve alto índice da realização do pré-natal. Há também prevalência de } \\
\text { gestações anteriores nessas adolescentes. Essa faixa etária predomina } \\
\text { meninas que nunca usaram métodos anticoncepcionais. Mais da metade das } \\
\text { adolescentes relatam que a gravidez foi planejada. }\end{array}$ \\
\hline $\begin{array}{l}\text { FRANÇA ACH, } \\
\text { et al., } 2013 .\end{array}$ & $\begin{array}{l}\text { Avaliar o perfil de gestantes adolescentes atendidas no Programa } \\
\text { da Saúde da Família (PSF) } 302 \text { da cidade de Aragarças, GO. }\end{array}$ & $\begin{array}{l}\text { Trata-se de um } \\
\text { estudo quantitativo e } \\
\text { qualitativo na } \\
\text { modalidade de estudo } \\
\text { observacional. }\end{array}$ & $\begin{array}{l}\text { A maioria das gestantes possuem mais de } 23 \text { anos e o nível socioeconômico } \\
\text { baixo, e tendo uma gravidez não planejada, podem estar propensas a } \\
\text { distúrbios afetivos. }\end{array}$ \\
\hline $\begin{array}{l}\text { SOUZA AXA, et } \\
\text { al., } 2012 .\end{array}$ & $\begin{array}{l}\text { Analisar as representações de adolescentes grávidas sobre a } \\
\text { gravidez na adolescência, com base na Teoria das } \\
\text { Representações Sociais. }\end{array}$ & Pesquisa qualitativa. & $\begin{array}{l}\text { Na adolescente grávida, o desejo de tornar-se independente, mulher e adulta } \\
\text { é confrontado com a angústia pela perda de proteção, revelando as fragilidades } \\
\text { próprias de sua fase ainda em desenvolvimento biopsíquico-afetivo. }\end{array}$ \\
\hline
\end{tabular}




\begin{tabular}{|c|c|c|c|}
\hline Autores e Ano & Objetivos do estudo & Perfil metodológico & Principais resultados \\
\hline $\begin{array}{l}\text { TAVARES KO, et } \\
\quad \text { al., } 2012 .\end{array}$ & $\begin{array}{l}\text { Traçar o perfil de puérperas adolescentes atendidas em um } \\
\text { Hospital Ensino do Sul do país }\end{array}$ & $\begin{array}{l}\text { Pesquisa } \\
\text { observacional, } \\
\text { retrospectiva, } \\
\text { transversal. }\end{array}$ & $\begin{array}{l}\text { A maioria das puérperas adolescentes têm ensino fundamental incompleto, } \\
\text { renda mensal baixa, não exercem atividade remunerada, tiveram início } \\
\text { precoce da vida sexual, alta taxa de acompanhamento pré natal, mas muitas } \\
\text { com início tardio, a maioria primigesta, e com partos vaginais. }\end{array}$ \\
\hline $\begin{array}{l}\text { SANTOS MMAS, } \\
\quad \text { et al., } 2012 .\end{array}$ & $\begin{array}{l}\text { Identificar associação entre estado nutricional pré-gestacional, } \\
\text { ganho ponderal materno e condições do pré-natal com os } \\
\text { desfechos prematuridade e baixo peso ao nascer (BPN) em filhos } \\
\text { de mães adolescentes. }\end{array}$ & $\begin{array}{l}\text { Trata-se de um } \\
\text { estudo quantitativo na } \\
\text { modalidade de estudo } \\
\text { transversal. }\end{array}$ & $\begin{array}{l}\text { As chances de BPN e de prematuridade reduziram quando a adolescente } \\
\text { recebeu } 6 \text { ou mais consultas de PN. }\end{array}$ \\
\hline $\begin{array}{l}\text { FURTADO EZL, } \\
\text { et al., } 2016 .\end{array}$ & $\begin{array}{l}\text { Identificar os fatores que interferem no acesso de adolescentes e } \\
\text { jovens à assistência ao parto na região Nordeste do Brasil. }\end{array}$ & $\begin{array}{l}\text { Estudo transversal e } \\
\text { de campo. }\end{array}$ & $\begin{array}{l}\text { Metade das adolescentes e jovens entrevistadas não foi orientada sobre o local } \\
\text { que deveria procurar para o parto, e entre aquelas que foram orientadas, } 23,5 \% \\
\text { não realizaram o parto no serviço de saúde indicado. }\end{array}$ \\
\hline $\begin{array}{l}\text { BACELAR EB, et } \\
\text { al., } 2017 .\end{array}$ & $\begin{array}{l}\text { Analisar possíveis associações entre Síndrome Hipertensiva } \\
\text { Específica da Gestação (SHEG) e características } \\
\text { sociodemográficas, do pré-natal e do parto de mães adolescentes } \\
\text { e adultas jovens. }\end{array}$ & $\begin{array}{l}\text { Trata-se de um } \\
\text { estudo quantitativo na } \\
\text { modalidade de estudo } \\
\quad \text { transversal. }\end{array}$ & $\begin{array}{l}\text { Do total de } 2.960 \text { adolescentes e adultas jovens, } 135(4,6 \%) \text { desenvolveram } \\
\text { SHEG. Puérperas sem companheiro apresentaram chance } 50 \% \text { maior de } \\
\text { apresentar esta patologia. }\end{array}$ \\
\hline $\begin{array}{l}\text { PAIVA AM, et } \\
\text { al.,2020. }\end{array}$ & $\begin{array}{l}\text { Analisar os fatores de risco para a gravidez em adolescentes na } \\
\text { faixa etária de } 10 \text { a } 19 \text { anos na Unidade de Referência } \\
\text { Especializada Materno Infantil (UREMIA); verificar a utilização de } \\
\text { métodos contraceptivos na população acima especificada; } \\
\text { verificar o perfil socioeconômico da família das adolescentes. }\end{array}$ & $\begin{array}{l}\text { Estudo quantitativo } \\
\text { descritivo } \\
\text { observacional. }\end{array}$ & $\begin{array}{l}30,4 \% \text { tiveram a sexarca aos } 13 \text { e } 14 \text { anos, } 39,3 \% \text { são solteiras, } 72,7 \% \text { têm } \\
\text { conhecimento sobre IST's sendo que } 54,1 \% \text { destas orientações foram } \\
\text { passadas principalmente na escola, } 54,6 \% \text { usam meios contraceptivos. } 48,8 \% \\
\text { possuem ensino fundamental incompleto e } 51,5 \% \text { das jovens têm uma renda } \\
\text { familiar de até um salário mínimo. }\end{array}$ \\
\hline $\begin{array}{l}\text { FREITAS JESM, } \\
\text { et al., } 2019 .\end{array}$ & $\begin{array}{l}\text { Relacionar características socioeconômicas, uso do fumo, do } \\
\text { álcool e do comportamento sexual entre as adolescentes } \\
\text { primíparas, primigestas e nulíparas. }\end{array}$ & $\begin{array}{l}\text { Estudo transversal } \\
\text { controlado, } \\
\text { analítico. }\end{array}$ & $\begin{array}{l}\text { As primíparas e primigestas apresentaram maiores frequências de vivência } \\
\text { com companheiro, maior percentual sem ocupação, menor número de } \\
\text { estudantes, maior frequência de mães com baixa escolaridade, pertencentes } \\
\text { aos estratos econômicos C, D ou E. As primíparas apresentaram frequências } \\
\text { mais elevadas de fumantes ou ex-fumantes, uso nocivo de álcool, não } \\
\text { utilização de preservativos na primeira relação e de relação nos últimos } 12 \\
\text { meses, porém com maior uso do preservativo. }\end{array}$ \\
\hline $\begin{array}{l}\text { HONÓRIO ME, } \\
\text { et al., } 2019 .\end{array}$ & $\begin{array}{l}\text { Apreender as percepções da mãe adolescente e da equipe de } \\
\text { enfermagem face ao cuidado ao filho prematuro hospitalizado na } \\
\text { unidade neonatal. }\end{array}$ & $\begin{array}{l}\text { Estudo qualitativo } \\
\text { com triangulação de } \\
\text { dados. }\end{array}$ & $\begin{array}{l}\text { A análise possibilitou a construção de oito categorias: Gravidez indesejada; } \\
\text { Sentindo se incapaz; Medo e culpa frente o nascimento prematuro; Confiança } \\
\text { na equipe; Tornando-se mãe, Dificuldade de trabalhar com mães } \\
\text { adolescentes, Imaturidade para ser mãe, Desenvolvendo responsabilidade. }\end{array}$ \\
\hline $\begin{array}{l}\text { CAMPOS CAT, } \\
\text { et al., } 2019 .\end{array}$ & $\begin{array}{l}\text { Investigar a percepção de adolescentes grávidas sobre gestação } \\
\text { precoce. }\end{array}$ & $\begin{array}{l}\text { Estudo qualitativo de } \\
\text { caráter exploratório. }\end{array}$ & $\begin{array}{l}\text { O estudo resultou em três categorias: Percepções de adolescentes grávidas } \\
\text { sobre ser mãe jovem, mudanças no cotidiano, e preconceitos e dificuldades } \\
\text { em ser mãe adolescente. Os resultados apontaram que dentre as onze } \\
\text { entrevistadas, a maioria se declarou parda e com ensino médio incompleto. }\end{array}$ \\
\hline
\end{tabular}

Fonte: Gonzaga PGA, et al., 2021. 


\section{DISCUSSÃO}

\section{Gravidez na adolescência: epidemiologia no Brasil}

A gravidez na adolescência abrange, segundo Gallo JHS (2011), a gestação ocorrida entre as idade de 10 e 19 anos completos, desde os anos de 1970. Devido a imaturidade biopsicossocial da jovem, a gravidez compreendida durante a adolescência é classificada como uma gestação de alto risco, podendo acarretar consequências graves no âmbito clínico, biológico e comportamental, além das consequências para o concepto.

As taxas de gestação na adolescência vêm ganhando forma considerável ao longo dos anos. França ACH, et al. (2013) expõem que, em 1998, 25\% dos partos realizados foram em adolescentes, logo após alguns anos estudos publicados revelaram que essa taxa subiu para $29,5 \%$, com ênfase nas regiões de Salvador, Porto Alegre e Rio de Janeiro.

Ribeiro CPS, et al. (2016) relatam que nos anos de 2011 e 2012 a prevalência de adolescentes grávidas aumentou cerca de $84 \%$ e que $18 \%$ dessas jovens teriam idades entre 15 e 19 anos sendo que estas já estariam na sua segunda gestação. Segundo os articulistas, no Brasil é observado que, em média, cerca de 700 mil adolescentes se tornam mães anualmente, e $2 \%$ (14.000 dessas jovens possuem de 10 a 14 anos).

Já Taborda JA, et al. (2014) perceberam que no âmbito mundial, aproximadamente, 7,3 milhões de jovens menores de 18 anos dão à luz todos os anos. Destas, 2 milhões ou 19,3\% possuem idades entre 10 e 15 anos e conclui dizendo que a taxa de fecundidade entre adolescentes é alarmante, em comparação com o resto da população.

O último estudo que abordou a epidemiologia da gestação na adolescência, segundo a pesquisa realizada, foi o de Bacelar EB, et al. (2017). De acordo com suas colocações e mesmo considerando que a taxa de fecundidade entre as mulheres brasileiras vem caindo consideravelmente, as adolescentes entre 15 e 19 anos representam cerca de $23 \%$ da fecundidade nacional. Dessa forma, percebe-se que a gravidez na adolescência vem se tornando um caso de calamidade pública no país, principalmente se consideradas as questões de vulnerabilidade nas quais essas jovens estão ou estarão expostas.

\section{Causas da gravidez na adolescência}

Taborda JA, et al. (2014), relatam que a ocorrência de gravidez na adolescência está em grande parte ligada ao nível socioeconômico das adolescentes, pois as classes econômicas mais vulneráveis apresentam um índice mais elevado desse evento. Além disso, parece ser um consenso o fato de que a gravidez indesejada é causada pela falta de conhecimento a respeito de métodos contraceptivos e de que quanto mais precoce se inicia a atividade sexual, mais chances terão essas adolescentes de uma possível gravidez. Assim, pode-se observar que o grau de escolaridade das adolescentes está diretamente ligado às chances do uso de preservativos tanto na primeira relação como nas relações subsequentes.

Dados do estudo de Carmo SS, et al. (2014) corroboram com essa relação, pois indica que a baixa escolaridade está diretamente relacionada com a gravidez na adolescência. Nesse mesmo sentido, estudos de Campos CAT, et al. (2019) demonstram que a gravidez na adolescência é multicausal, e, por isso, os fatores que levam as jovens a engravidarem estão amplamente relacionados a fatores sociais, biológicos psicológicos, familiares e a métodos contraceptivos usados de maneira incorreta pelos adolescentes.

No entanto, Ribeiro CPS, et al. (2016) mostram que mesmo com o aumento do acesso às informações sobre prevenção e sexualidade, os índices de doenças sexualmente transmissíveis e de gravidez não planejada não estão sofrendo redução. Dessa forma, os autores reforçam que é importante avaliar o conhecimento com o estímulo de práticas saudáveis a partir de estratégias que promovam a autorreflexão dos jovens em situações mais vulneráveis para que consigam identificar o que o leva a multiplicação dos fatores de risco e de vulnerabilidade relacionados à sexualidade.

Em consonância, estudos de Santos MMAS, et al. (2012) revelam que a maior parte das adolescentes não fazem uso de métodos contraceptivos, mesmo sabendo que existem e onde encontrá-los. Confirma, assim, 
que o conhecimento em relação a contracepção não garante eficácia em relação à prevenção da gravidez, apontando questões em relação à excelência das ações voltadas para a sexualidade e a saúde reprodutiva dos adolescentes.

França $\mathrm{ACH}$, et al. (2013) reportaram o fato de que antes as adolescentes iniciam a vida sexual em média com 15 anos, porém a redução desse início médio da vida sexual, a queda da idade da menarca, o pouco conhecimento do processo de reprodução, a falta de informação sobre métodos contraceptivos, o pouco acesso a esses métodos e seu uso inadequado, constituem causas importantes para a ocorrência da gravidez na adolescência.

Portanto, para Kudlowiez S e Kafrouni R (2014) a gravidez na adolescência, a partir de uma visão social e histórica, deve-se levar em consideração, entre outros aspectos, a condição social das jovens e futuras mães. Pois esse fenômeno é uma questão intrincada, sendo necessário problematizar os discursos homogêneos em relação ao tema, considerando a situação social e econômica dos jovens brasileiros.

\section{Riscos da gestação na adolescência}

Segundo Taborda, et al., (2014), a prevalência de morte ligada à gravidez precoce (entre 15 e 19 anos) e intraparto é duas vezes maior em comparação a mulheres de 20 anos. Quando observada em menores de 15 anos, esse risco sobe em cinco vezes. Ainda segundo os autores, essa temática já representa, no Brasil, um problema de saúde pública, pois as repercussões, como anemia, pré-eclampsia, síndromes hipertensivas e restrição de crescimento fetal, além de complicações decorrentes de abortos ou pela escassez de assistência regular e de qualidade, podem estar presentes em gravidez com gestante menor de 19 anos. A associação pertinente realizada pelos autores Ribeiro CPS, et al. (2016), diz que a gestação precoce poderá resultar em prematuridade e riscos de mortalidade materna e infantil.

Santos MMAS, et al. (2012), discorrem sobre a controvérsia, presente na literatura. Apresentam estudos, os quais defendem que o principal fator de risco relacionado a complicações gestacionais e pós-natais são a imaturidade (tanto física, quanto biológica) da adolescente. Já outros apontam que o grau dessas intercorrências dizem respeito ao nível de vulnerabilidade a que a jovem está inserida. Relatam também sobre a possibilidade de uma competição materno-fetal por nutrientes, uma vez que o processo de competição entre mãe e concepto, já que ambos estão em fase de desenvolvimento. Esse processo poderá apresentar riscos, como baixo peso ao nascer, deficiências de micronutrientes e restrição do crescimento intrauterino, refletindo negativamente na evolução da gestação, bem como no desenvolvimento fetal.

Bacelar EB, et al. (2017), especificam que a Síndrome da Gestação Hipertensiva Específica (SGHS) é considerada uma das principais causas de morte materna e neonatal. Apresentam estudos que comprovam que nos países desenvolvidos, a incidência varia de 2 a $8 \%$ das gestações, enquanto no Brasil pode chegar a mais de $10 \%$, sendo considerada a primeira causa de morte materna, apresentando alto índice de morbimortalidade perinatal. Os autores relatam que a etiologia da SGHS não é bem conhecida, mas destacam como fator de risco para sua ocorrência a gravidez na adolescência. Justifica-se que as adolescentes grávidas são mais vulneráveis à ocorrência de SGHS quando comparadas aos adultos jovens, pelo fato de apresentarem imaturidade de órgãos e sistemas, bem como desinformação e dificuldade de acesso ao Sistema de Saúde.

Honório ME, et al. (2019) discorreram quanto à crise individual que a gravidez precoce gera na mãe adolescente. A incerteza do mundo novo que há de vir, as alterações físicas marcantes, a fragilidade dos vínculos sociais após a gestação pode gerar reflexos psicológicos que resultam em alterações físicas gestacionais. O risco de parto prematuro e morbidade fetal é relativamente alto nesses casos.

Sobre os riscos da gravidez na adolescência, dos 16 artigos escolhidos para a revisão, apenas cinco discorreram sobre a temática. Nesses artigos, identificou-se abordagens superficiais e com explicações breves sobre os riscos. Portanto, os riscos da gravidez na adolescência não estão sendo abordados de forma integral e eficaz para contribuição de estudos científicos desses casos. Nesse sentido, abordagens sobre o assunto devem ser estimuladas, visando um enfoque na compreensão dos reflexos físicos na gestante jovem. 


\section{Consequências psicossociais de uma gestação precoce}

A gravidez na adolescência, se associa a diversas mudanças físicas, mas há também diversos problemas relacionados ao psicológico dessas adolescentes. Uma vez que transformações no corpo causam a diminuição da autoestima, há aumento de casos de depressão, a instabilidade de um relacionamento, a evasão escolar e o aumento da reincidência de gravidez, podem vir a trazer consequências psicossociais. De acordo com estudos, uma parcela grande das adolescentes grávidas são de baixo nível socioeconômico e não planejaram sua gestação, o que relaciona os distúrbios afetivos encontrados na maioria delas (FRANÇA $\mathrm{ACH}$, et al., 2013).

Segundo França $\mathrm{ACH}$, et al. (2013), as adolescentes tendem a estabelecer relacionamentos com homens adolescentes sem atividade ocupacional, o que é um risco social e torna a vida dos pais e do filho precárias, uma vez que a maioria das mães também não exercem atividade remunerada. Ainda nesse estudo, é notável uma grande evasão escolar, além de um alto índice de uso de drogas por essas adolescentes. A proporção de problemas emocionais nessas mães teve porcentagem alta, corroborando com as teorias de que a gravidez acarreta mudanças no corpo da mulher que podem levar à ansiedade, depressão e até ideação suicida.

Estudos mostram que o maior sentimento entre as adolescentes que engravidam é a sensação de perda, tanto de liberdade quanto do apoio da família, parceiro e amigos. Além disso, há o medo e a angústia, relacionados a inexperiência dessas adolescentes, e também a condições socioeconômicas. Por outro lado, evidencia-se sentimentos de amor, desejo, realização, crescimento e amadurecimento, em função do novo papel social que as adolescentes assumem como futuras mães (SOUZA AXA, et al. 2012). Com a grande alteração no corpo dessas adolescentes, e também no psicológico das mesmas, é essencial uma rede de assistência que promova além de informações de planejamento familiar, ações em saúde visando os riscos sociais que a gravidez na adolescência acarreta (FREITAS JESM, et al. 2019)

Entre as adolescentes com uma gravidez indesejada, a taxa de aborto ilegal é elevada, e sua maior consequência é o procedimento feito de maneira inadequada que leva à internação e muitas consequências físicas e também psicológicas. Nos casos de internação por aborto, a adolescente tem direito à proteção do sigilo e dos cuidados em saúde, e o profissional não pode notificar a autoridade policial, judicial ou o Ministério Público (JUNIOR EVS, et al. 2018). Um estudo em 2011 na cidade de Teresina (PI), teve como resultado os relatos das adolescentes que disseram que as complicações do aborto foram consequência da precariedade da assistência médica às mulheres, com relação a maus-tratos, denúncias e preconceito por parte dos profissionais, o que mostra a deficiência desse atendimento e a necessidade de capacitação e sensibilização desses profissionais de saúde (NUNES MD, et al. 2013).

\section{CONSIDERAÇÕES FINAIS}

A gravidez na adolescência tem como causa principal a falta de conhecimento sobre os métodos contraceptivos. Isso pode ter associação com a situação de vulnerabilidade socioeconômica na qual essa adolescente está inserida. Sobre os riscos, a mãe e o concepto estão predispostos à alterações físicas, como restrição de crescimento fetal, síndrome hipertensiva, pré-eclâmpsia, entre outros. Já quanto às consequências psicossociais, a depressão e os reflexos na autoestima da jovem foram os mais ressaltados. O presente estudo possui grande relevância científica, pois poderá contribuir para que profissionais da saúde possam se conscientizar de que a raiz do problema ainda está na promoção de saúde que deve ser fortalecida e realizada com foco ainda maior nas jovens mais vulneráveis.

\section{REFERÊNCIAS}

1. BACELAR EB, et al. Fatores associados à síndrome hipertensiva específica da gestação em puérperas adolescentes e adultas jovens da região Nordeste do Brasil: análise múltipla em modelos hierárquicos. Rev Bras de Saúde Materno Infantil, 2017; 17(4): 683-691.

2. CABRAL ALB, et al. A gravidez na adolescência e seus riscos associados:revisão de literatura. Braz. J. Hea. Rev, 2020; 3(6): 19647-19650. 
3. CABRAL CS, BRANDÃO ER. Gravidez na adolescência, iniciação sexual e gênero: perspectivas em disputa.Cad. Saúde Pública, 2020; 36(8): 1-5.

4. CAMPOS CAT, et al. Percepção de adolescentes grávidas sobre a gestação precoce. Revista Eletrônica Acervo Saúde, 2019; 22: 1-9.

5. CARMO SS, et al. Análise quantitativa sobre gravidez na adolescência em um município mineiro. Cogitare Enferm, 2014; 19(4): 801-807.

6. CASTRO ABC, et al. Gênero, patriarcado, divisão sexual do trabalho e a força de trabalho feminina as sociedade capitalista. VI seminário CETROS. 2018; 2(6): 2-15.

7. ERCOLE FF, et al. Revisão integrativa versus sistemática. Rev Min Enferm, 2014; 18(1): 10.

8. FARIA GRB, MAGALHÃES CMC. Correlações entre maternidade na adolescência e bebê prematuro e de baixo peso: uma revisão integrativa.Mudanças - Psicologia da Saúde, 2020; 28(1): 63-73.

9. FRANÇA ACH, et al. Gestação precoce e reincidência de gestações em adolescentes e mulheres de uma unidade de estratégia de saúde da Família (ESF 302). Rev APS, 2013; 16(2): 129-135.

10. FREITAS JESM, et al. Fatores Sociais e de saúde relacionados à gravidez na adolescência. Revista Eletrônica Acervo Saúde, 2019; 30: 1-9.

11. FREITAS MVP, SANTOS FR. Gravidez na adolescência: um problema de saúde pública no Brasil. Revista da Jornada de Pós-graduação e Pesquisa, 2020; 16(16): 227-231.

12. FURTADO EZL, et al. Acesso à assistência ao parto de adolescentes e jovens na região Nordeste do Brasil. Rev Saúde Pública, 2016; 50:23.

13. GALLO JHS. Gravidez na adolescência: a idade materna, consequências e repercussões. Rev. Bioét, 2011; 19(1):179195.

14. HONÓRIO ME, et al. O processo do cuidar do bebê prematuro: percepções da mãe adolescente e equipe de enfermagem. Revista Eletrônica Acervo Saúde, 2019; 11(7): 1-9.

15. IACOBELLI S, et al. Obstetric and neonatal outcomes of adolescent primiparous singleton pregnancies: a cohort study in the South of Reunion Island, Indian Ocean. J Matern Fetal Neonatal Med, 2012; 25(12): 2591-6.

16. JUNIOR EVS, et al. Dilemas bioéticos na assistência médica às gestantes adolescentes. Rev. Bioét, 2018 ; $26(1)$ : 8794.

17. KUDLOWIEZ S, KAFROUNI R. Gravidez na Adolescência e Construção de um Projeto de Vida. Rev Ele PUCRS, 2014; 45(2): 228-238.

18. MARANHÃO TA, et al. Atitudes e reações familiares e sociais diante da gravidez na adolescência. Rev enferm UFPE on line, 2018; 12(4): 840-848.

19. MINISTÉRIO DA SAÚDE. Secretaria de Atenção à Saúde, Departamento de Ações Programáticas e Estratégicas. Proteger e cuidar da saúde de adolescentes na Atenção Básica [recurso eletrônico].Brasília - DF, Brasil, 2017. 234 p.:il. Disponível em: https://bvsms.saude.gov.br/bvs/publicacoes/proteger_cuidar_adolescentes_atencao_basica.pdf. Acessado em 10 de junho de 2021.

20. MIURA PO, et al. O desamparo vivenciado por mães adolescentes e adolescentes grávidas acolhidas institucionalmente. Ciência e Saúde Coletiva, 2018; 23(5): 1601-1610.

21. NOVELLINO MSF. Um estudo sobre as mães adolescentes brasileiras. Physis Revista de Saúde Coletiva, 2011; 21(1): 299-318.

22. NUNES MD, et al. Histórias de aborto provocado entre adolescentes em Teresina, Piauí, Brasil. Ciência e Saúde Coletiva, 2013; 18(8): 2311-231.

23. OYAMADA LH, et al. Gravidez na adolescência e o risco para a gestante. Brazilian Journal of Surgery and Clinical Research, 2014; 6(2): 38-45.

24. PAIVA AM, et al. Fatores que propiciam a gravidez na adolescência em uma unidade de referência especializada materno infantil na região Norte do Brasil: um estudo piloto. Revista Eletrônica Acervo Saúde, 2020; 49: 1-9.

25. PINHEIRO YT, et al. Fatores associados à gravidez em adolescentes de u município do nordeste do Brasil. Cad. Saúde Colet, 2019; 27(4): 363-367.

26. RIBEIRO CPS, et al. Percepção de adolescentes escolares sobre transformações corporais, gravidez e caderneta de saúde do adolescente. Rev Cubana de Enfermaria, 2016; 32(1): 27-36.

27. SANTOS MMAS, et al. Estado nutricional pré-gestacional, ganho de peso materno, condições da assistência pré-natal e desfechos perinatais adversos entre puérperas adolescentes. Rev Bras Epidemiol, 2012; 15(1): 143-154.

28. SOCIEDADE BRASILEIRA DE PEDIATRIA (SBP). Consulta do adolescente: abordagem clínica, orientações éticas e legais como instrumentos ao pediatra. Departamento Científico de Adolescência, 2019. Disponível em: https://www.sbp.com.br/fileadmin/user_upload/21512c-MO_-_ConsultaAdolescente_-_abordClinica_orientEticas.pdf. Acessado em 10 de junho de 2021.

29. SOUZA AXA, et al. Representações sociais de adolescentes grávidas sobre a gravidez na adolescência. Psicologia e Sociedade, 2012; 24(3): 588-596.

30. SOUZA MT, et al. Revisão integrativa: o que é e como fazer? Einstein, 2010; 8(1): 102-106.

31. TABORDA JA, et al. Consequências da gravidez na adolescência para as meninas considerando-se as diferenças socioeconômicas entre elas. Cad. Saúde Colet, 2014; 22(1): 16-24.

32. TAVARES KO, et al. Perfil de puérperas adolescentes atendidas em um hospital ensino do sul do país. Acta Scientiarum Health Sciences, 2012; 34(1): 9-15. 